\title{
INSIDE THE USCAP JOURNALS
}

\section{MODERN PATHOLOGY}

\section{Prognostic significance of evaluation of lymph node metastases \\ https://doi.org/10.1038/s41379-021-00871-1}
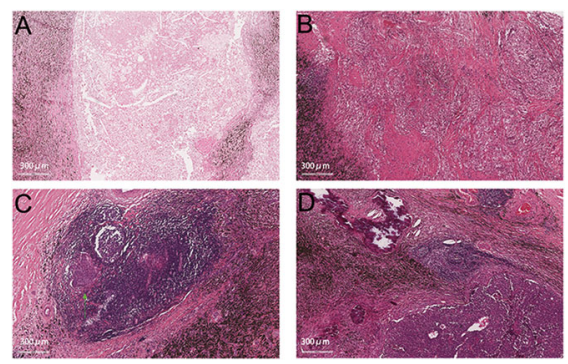

Little is known of the prognostic significance of posttreatment pathologic evaluation of metastatic lymph node responses. Liu et al. report the findings of a retrospective study of 336 patients with non-small-cell lung cancer (NSCLC) who underwent neoadjuvant therapy. Using the standards of the International Association for the Study of Lung Cancer, primary and lymph node metastases (LNM) were pathologically assessed, and the relationship between overall survival (OS) and disease-free survival (DFS) in each was analyzed. Pathologic assessment identified LNM in 208/336 patients. On multivariable analysis, residual viable tumor (\% RVT) of the primary tumor $\leq 12 \%$ was an independent prognostic factor for improved OS, whereas an RVT of LNM $\leq 8 \%$ was an independent prognostic factor for increased DFS. Thus, while assessment of the primary tumor is prognostically meaningful, assessment of the lymph nodes is equally important and significant.

\section{Clinicopathology and genomics of NEC of the head and neck} https://doi.org/10.1038/s41379-021-00869-9
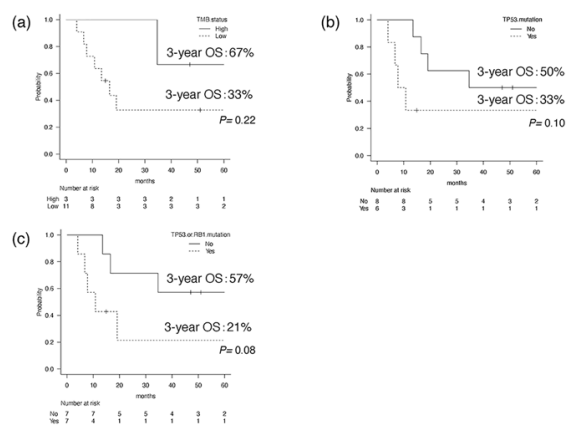

Using a retrospective analysis of 27 patients with poorly differentiated neuroendocrine carcinoma (NEC) of the head and neck, Ohmoto et al. explored this rare malignancy that comprises $0.3 \%$ of head and neck cancers. The most common tumor locations were the paranasal sinus (33\%) and the oropharynx (19\%), with $81 \%$ of patients showing locally advanced disease. The 3-year overall survival of all patients was 39\%, but it was 53\% in the 17 patients with locally advanced disease who received multimodal curative treatment. Targeted-capture sequencing of 523 cancer-related genes revealed lower prevalence of mutations in TP53 and RB1 as compared with that in smallcell lung carcinoma, as well as FGFR3-TACC3 gene fusion, deleterious gene variants in NOTCH1, and several genes encoding components of the PI3K/AKT/mTOR pathway. Patients with locally advanced disease had favorable outcomes, despite the presence of aggressive pathological features.

\section{LABORATORY INVESTIGATION}

\section{Targeting rRNA biogenesis as} therapeutics for metastatic disease https://doi.org/10.1038/s41374-021-00642-1

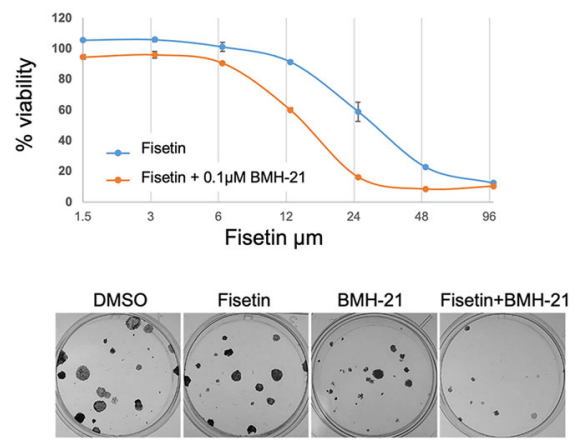

Kammerud et al. explored the role of fisetin-a nutraceutical flavanol from the flavonoid group of polyphenols-in cancer progression. They found that fisetin negatively impacts several signaling pathways known to support tumor progression. One way that it does this is through its ability to enter the nucleolus. In breast tumor cells treated with fisetin, a 20-30\% decrease in nuclear abundance per cell correlated with a 30-60\% downregulation of RNA Pol I transcription activity as well as a $50-70 \%$ reduction in nascent rRNA synthesis. The decrease in RNA Pol I activity and ribosomal RNA (rRNA) biogenesis is apparently due to the negative influence of fisetin on the MAPK/ERK pathway. In an animal model, the combination of fisetin and BMH-21 (an RNA Pol I inhibitor) acted synergistically to elicit a $60 \%$ decrease in lung 
colonization of breast cancer cells. rRNA biogenesis is a clear target for possible therapeutics for metastatic disease.

\section{Development of hEM3, a model of endometrium- related malignancy} https://doi.org/10.1038/s41374-02100624-3

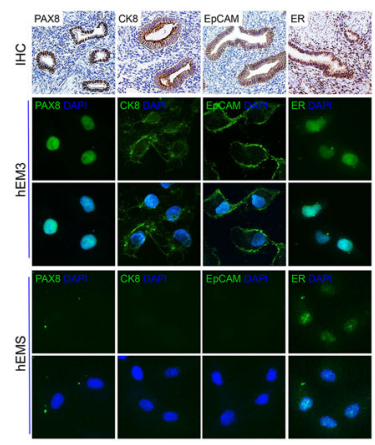

Endometrium-related malignancies claim more than 13,000 women's lives annually in the United States, and developing in vitro models to study both the "normal" endometrial epithelial cells and their malignant counterparts is crucial for studying the pathogenesis of the diseases. Park et al. developed a human endometrial epithelial cell line, hEM3, through immortalization and clonal selection. Just as in the endometrial epithelium, hEM3 expresses PAX8, EpCAM, cytokeratin 7.8, and ER. As importantly, hEM3 cells are not tumorigenic when transplanted into immunocompromised mice. Gene editing of the cell line has been shown to be stable, with a knockout of ARID1A, a tumor suppressor gene, being followed by successful targeting of affected cells with HDAC and PARP inhibitors. The group proposes that hEM3 may be an effective tool not only for studying the pathology of endometriumrelated disease but also as a tool for identifying and exploring precision therapeutics.

\section{nature.com/pathology}

Determining the value of repeated WGS over time in metastatic cancer

\section{patients}

While there are data to support genomic profiling for the identification of treatment options for metastatic cancer patients, little is known about the benefits of repeating the testing over time. Using a pool of 231 adult patients with a variety of metastatic solid malignancies, van de Haar et al. analyzed whole-genome sequencing (WGS) data of 250 biopsy pairs. Patients received one or more line of therapy between the two biopsies with a median interval of 6.4 months between the two. Standard-of-care (SOC) genomic biomarkers and biomarkers for clinical trial enrollment could be identified in $23 \%$ and $72 \%$ of biopsies, respectively, and $99 \%$ of paired biopsies showed concordance of SOC biopsies between the two. When the investigators sought genes targeted for smallmolecule inhibitors or hormonal therapies, $21 \%$ and 22\% of cases, respectively, were shown to evolve between

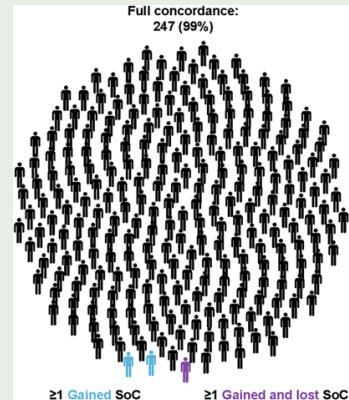
$\begin{array}{cc}\geq 1 \text { Gained SoC } & \begin{array}{c}21 \text { Gained and lost SoC } \\ \text { treatment indication: } \\ 2(0.8 \%)\end{array} \\ \text { treatment indication: } \\ 1(0.4 \%)\end{array}$ pairs. Most illuminating was the observation that in $91 \%$ of patients a second WGS analysis did not identify additional biomarkers for clinical trial enrollment, indicating little support for repeated WGS for optimal selection of treatment options. Disease progression, the authors suggest, is not driven primarily by ongoing accumulation of fitness-promoting genomic driver alterations that continuously increase the aggressiveness of the disease. Repeat biopsy may be more important to identify mutations resulting in resistance to treatment.

Nature Medicine 2021;27:1553-1563; https://doi.org/10.1038/s41591-021-01448-w

\section{Exploring the accumulation of somatic mutations in normal tissue as we}

age

Acknowledging that both aging and disease are associated with the accumulation of somatic mutations even in normal tissues, Li et al. performed genomic analysis of 1737 morphologically normal tissue biopsies of nine organs from five donors to look for patterns. They found that the extent of mutation accumulation as well as their clonal expansion differed greatly in different organs. Endogenous mutational processes with the SBS1 and SBS5 mutational signatures are ubiquitous but have different relative activities, whereas exogenous mutational processes operate in multiple tissues of the

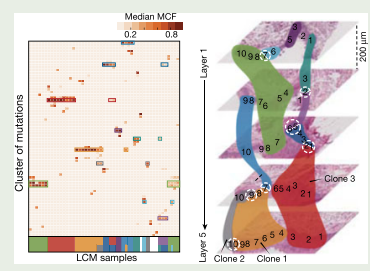
same donor. Somatic copy number alterations are found in the esophagus and gastric cardia and rarely in any other tissue. In these two tissues, it was also possible to see clonal expansion of somatic clones to hundreds of micrometers, whereas in tissues such as colon, rectum, and duodenum, somatic clones were microscopic-possibly restricted by the architecture of the tissues themselves - and therefore evolved independently. With expansion of the sample size needed for further validation, the group was able to develop maps of somatic mutations within individuals in their sample, which has implications for tumorigenesis and progress as well as diagnostics and therapeutics.

Nature, published online 25 August 2021; https://doi.org/10.1038/s41586-021-03836-1

Reviews written by Emma Judson, Ph.D.

For a Chinese version of Inside the USCAP Journals, see the Supplementary Material.

Chinese translation by Cheng Jin, Jinxuan Xiahou, Xinyue Li, Hui Chen, Yuli Wang, Yongyu Yang, and section editor Jian Wu; Fudan University School of Basic Medical Sciences.

Supplementary Information The online version contains Supplementary Material available at https://doi.org/10. 1038/s41374-021-00000-0. 